\title{
GEORREFERENCIACIÓN DE LAS ESTACIONES SISMOLÓGICAS Y ACELEROGRÁFICAS DEL OBSERVATORIO SISMOLÓGICO DE LA UNIVERSIDAD DEL QUINDÍO IMPLEMENTANDO EL SISTEMA DE POSICIONAMIENTO GNSS
}

\author{
GEOREFERENCING OF THE SEISMOLOGICAL AND ACCELEROGRAPHIC \\ STATIONS OF THE SEISMO-LOGICAL OBSERVATORY OF THE UNIVERSITY \\ OF QUINDIO IMPLEMENTING THE SYSTEM OF POSITIONING GNSS
}

\begin{abstract}
Viviana Ruiz Zorrilla ${ }^{1 *}$, Marisol Gómez Cano²
1. Facultad de Ingeniería, programa de Ingeniería Civil, Universidad del Quindío, e-mail: viviruzo@hotmail.com.

2. Facultad de Ingeniería, Observatorio Sismológico de la Universidad del Quindío, e-mail: solgc@uniquindio.edu.co.
\end{abstract}

*Correspondencia del Autor: Viviana Ruiz Zorrilla email: viviruzo@hotmail.com

\section{RESUMEN}

El Observatorio Sismológico de la Universidad del Quindío (OSQ) cuenta con 6 estaciones sismológicas (Bolívar y Peñas Blancas de banda ancha, Caicedonia y Peñas Blancas analógicas, Modín y Riberalta de tipo digital) y 3 estaciones acelerográficas (Uniquindio, Calarcá y Génova) distribuidas estratégicamente en el departamento del Quindío y el Valle del Cauca con los que se lleva a cabo el monitoreo de la actividad sísmica de la zona. Dichas estaciones se encuentran georreferenciadas, pero son datos tomados hace 20 años que no han sido referenciados usando equipos de alta precisión, por esta razón, el Sistema de Posicionamiento (GNSS) se implementa con ayuda de un software especializado en la compilación de los datos y por consiguiente se mejora la localización de los eventos sísmicos registrados en el (OSQ). Para la recolección de la información se programaron campañas de medición usando la herramienta de Software GNSS Planning Online que permite conocer el día y el horario en que existe mayor disponibilidad de satélites para obtener mayor precisión. Para el procesamiento de los datos, se utilizó el método de postproceso que permite corregir los errores sistemáticos que se presentan durante la captura de información en campo y posteriormente se realizó un ajuste con el software TopCon Tools para entregar resultados con el menor error posible. Como producto final de la investigación, se entrega al (OSQ), un catálogo con las coordenadas geográficas de las estaciones debidamente georreferenciadas y ubicadas en un mapa de la región dejando un insumo que favorece las actividades realizadas por esta dependencia.

Palabras claves: Georreferenciación, Sistema de posicionamiento GNSS, postproceso, posicionamiento diferencial o relativo.

Cómo citar:

Ruiz Zorrilla, V., \& Gómez Cano, M. (2018) GEORREFERENCIACIÓN DE LAS ESTACIONES SISMOLÓGICAS Y ACELEROGRÁFICAS DEL OBSERVATORIO SISMOLÓGICO DE LA UNIVERSIDAD DEL QUINDÍO IMPLEMENTANDO EL SISTEMA DE POSICIONAMIENTO GNSS Revista de Investigaciones Universidad del Quindío. Vol 30, pp.15-22.

Información del artículo: Recibido: 29 noviembre 2018; Aceptado: 28 diciembre 2018 


\begin{abstract}
The Seismological Observatory of the Universidad del Quindío (OSQ) has 6 seismological stations (Bolívar, Peñas Blancas broadband, Caicedonia, Modín, Rivera and Peñas Blancas analogous) and 3 accelerographic stations (Uniquindio, Calarcá and Génova), strategically distributed in the department of Quindío and Valle del Cauca, which carry out the monitoring of seismic activity in the area. These stations are not georeferenced, for this reason, the Positioning System (GNSS) is implemented using high-precision instruments with the help of specialized software to achieve greater accuracy in the compilation of data and therefore improve the location of seismic events recorded in the (OSQ). In order to collect the information, it was necessary to carry out measurement campaigns through the GNSS Planning Online Software, which indicates the days when satellites are most available to obtain greater precision. For the data processing, the post-processing method was used to correct the systematic errors that occur during the capture of information in the field and later an adjustment was made with the TopCon Tools software to deliver results with the least possible error. As a final product of the investigation, a catalogue with the geographic coordinates of the stations duly georeferenced and located in a map of the region is delivered to (OSQ), leaving an input that favors the activities carried out by this agency.
\end{abstract}

Keywords: Georeferencing, GNSS positioning system, post-processing, differential or relative positioning.

\section{INTRODUCCIÓN}

El Observatorio Sismológico de la Universidad del Quindío (OSQ) cuenta con seis estaciones sismológicas y tres estaciones acelerográficas ubicadas en los departamentos del Quindío y Valle del Cauca, cuyas coordenadas, con las que actualmente se trabaja para localizar los eventos sísmicos de la región, fueron calculadas manualmente después del terremoto de Armenia en 1999, sin ningún tipo de ajuste o proceso de ajuste o proceso geodésico que garantice su exactitud. Por tal motivo, surge la necesidad de implementar un sistema de posicionamiento GNSS mediante una técnica llamada post proceso realizando el correspondiente ajuste de la información, que permite obtener con mayor precisión la ubicación de los puntos de medición mejorando por consiguiente la localización de los eventos sísmicos registrados por el OSQ.

El presente artículo deja en evidencia las coordenadas de las estaciones sismológicas y acelerográficas del Observatorio Sismológico de la Universidad del Quindío de manera precisa y debidamente georreferenciadas y ajustadas logrando el menor error posible utilizando un GPS doble frecuencia de alta precisión, el software Topcon Tools y sistemas de información geográfica como Google Earth y ArcGIS.

\section{RED SISMOLÓGICA DEL OBSERVATO- RIO SISMOLÓGICO DE LA UNIVERSI- DAD DEL QUINDÍO (OSQ)}

El Observatorio Sismológico de la Universidad del Quindío, define las estaciones sismológicas como una serie de instrumentos acoplados dentro de un sistema de medida, que se encuentran distribuidas en una zona geográfica determinada con el objetivo principal de registrar la actividad sísmica de una región. Al conjunto de varias estaciones sismológicas se le conoce con el nombre de Red Sismológica.

La Red Sismológica Nacional de Colombia (RSNC) encargada de proporcionar información general sobre el funcionamiento y la metodología empleada para llevar a cabo el monitoreo de la actividad sísmica en el país, determinó la zona epicentral de la región después del evento ocurrido en Armenia el 25 de enero de 1999. Con dicha información se realizó la instalación de la red sismológica local la cual contó con 21 estaciones sismológicas y acelerográficas. La configuración original de la red ha sufrido varias modificaciones, llegando hasta la red actual que cuenta con estaciones acelerográficas autónomas, sismológicas análogas con telemetría y sismológicas de banda ancha portátiles, con centro de recepción en la Universidad del Quindío. 
El principal objetivo de este trabajo es implementar el sistema de posicionamiento (GNSS) para georreferenciar las estaciones de medición del (OSQ) de la Universidad del Quindío, mediante la planificación de campañas de medición para la recolección de datos en campo con GPS doble frecuencia de alta precisión, el procesamiento de la información aplicando el método de postproceso utilizando herramientas de software libre TopCon Tools (Topcon Tools, 2017) y establecer las características de georreferenciación en cada una de las estaciones sismológicas y acelerográficas del (OSQ) con el menor error posible.

\section{METODOLOGÍA}

Para el desarrollo de la georreferenciación de las estaciones de medición del OSQ, se propuso la metodología que se puede apreciar en la figura 1, la cual contiene un trabajo de campo para la debida recolección de información y posteriormente un procesamiento de datos realizado en oficina para dar cumplimiento con los objetivos planteados.

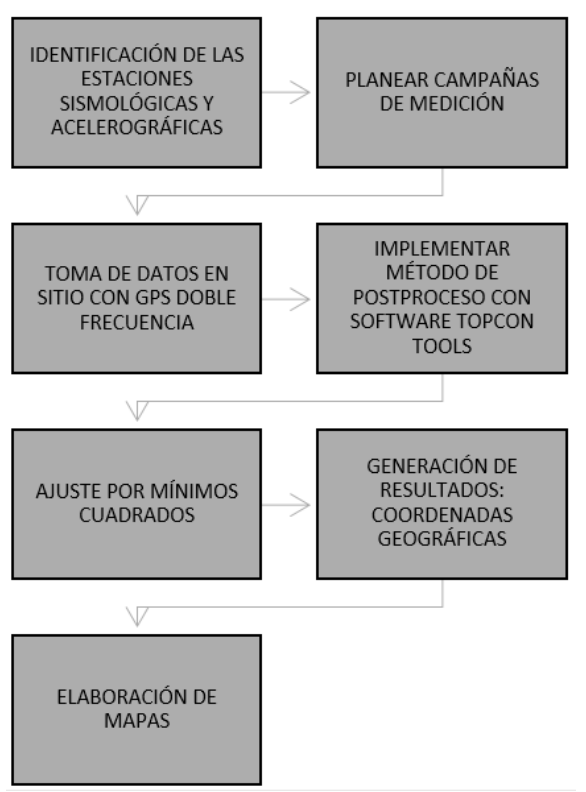

Figura 1. Esquema metodológico

Inicialmente, fue necesario realizar una breve descripción de las estaciones sismológicas y acelerográficas que en la actualidad pertenecen al OSQ, para conocer su ubicación y las coordenadas de referencia utilizadas para el proceso de localización de los eventos sísmicos.

Para la programación de las visitas a las diferentes estaciones del OSQ, se utilizó un programa llamado GNSS Planning Online, el cual permite determinar el número de satélites disponibles en las fechas y horas que se requieran, estableciendo un cronograma de las salidas de campo. Se decide realizar las respectivas mediciones con el navegador GPS South S82 doble frecuencia de alta precisión, el cual se ubicó en zonas con pocos obstáculos visuales y lo más cerca posible al sitio donde se encuentra el sensor de cada estación, puesto que se debía garantizar una mayor precisión. Para lograr los resultados esperados, el tiempo de exposición para desarrollar la toma de datos estaba entre 2 a 3 horas.

Al finalizar cada visita, con la información adquirida, era necesario conformar una base de datos de vectores entre los receptores de referencia ubicados en el OSQ llamado REF-UQ con coordenadas 4³3'20,66937' N, 75³9'37,39463'” $\mathrm{W}$ y en el aeropuerto El Edén llamado VEDE con coordenadas $4^{\circ} 27^{\prime} 37,07756^{\prime}$ N $, 75^{\circ} 45^{\prime} 55,58368^{\prime \prime} \mathrm{W}$, para asociarlos con los datos obtenidos en cada estación de medición. Posteriormente, se procesaron los datos utilizando el sistema de posicionamiento GNSS, el cual trabaja con más de un sistema de navegación satelital y el software TopCon Tools. Para este trabajo, se aplicó el método de postproceso el cual sirve para georreferenciar cada uno de los puntos y corregir los errores sistemáticos que se presentaron en la captura de información en campo y de esta manera mejorar la precisión de los resultados (Pachas, 2010).

Como el método de posicionamiento diferencial o relativo consiste en el uso de un punto con coordenadas previamente conocidas y un segundo punto el cual se desea obtener su ubicación (McCormac, 2008), en este caso se cuenta con dos estaciones de referencia conocidas de manera exacta que son la estación VEDE del Servicio Geológico Colombiano ubicada en el Aeropuerto El Edén en la Tebaida Quindío y la estación del Observatorio Sismológico de la Universidad del Quindío. 
Al tener la información procesada, fue necesario realizar un ajuste mediante el software TopCon Tools el cual utiliza el método de mínimos cuadrados para verificar los posibles errores y entregar resultados más precisos con el menor error posible (Ghilan, 2010).

Finalmente, utilizando el software ArcGIS (ArcGis, 2013) en el sistema de coordenadas geográficas UTM (Universal Transverse Mercator) con el WGS84 (World Geodetic System 84) en la zona 17 del hemisferio Sur y teniendo un mapa base, se marca la ubicación de los puntos georreferenciados (estaciones de medición), obteniendo un catálogo que incluye las coordenadas debidamente georreferenciadas y la respectiva ubicación en el espacio.

\section{ANÁLISIS Y DISCUSIÓN DE RESULTA- DOS}

Se presentan todos los resultados obtenidos con la ejecución de este proyecto, siguiendo la metodología propuesta y dando respuesta a los objetivos planteados.

\section{ESTACIÓN UNIQUINDÍO}

La estación sismológica Uniquindío está ubicada en el municipio de Armenia dentro de las instalaciones de la Universidad del Quindío, específicamente en la facultad de ingeniería, como se observa en la ilustración 1, contando con todos los equipos necesarios para la recopilación de información, que posteriormente es enviada al OSQ.

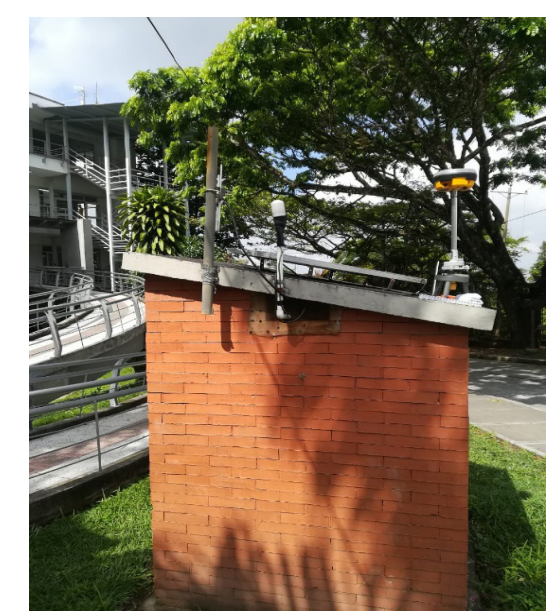

Ilustración 1.Estación UQ. Fuente: propia
La medición en este lugar, se realiza el día 22 de mayo de 2018, iniciando a las 9:47am y finalizando a las $12: 47 \mathrm{pm}$, donde se determina una altura instrumental de $2.49 \mathrm{~m}$ medidos desde el suelo. Finalmente, al procesar los datos obtenidos y realizar el respectivo ajuste, se tienen las siguientes coordenadas georreferenciadas: 4³3'21,83385'N y 75³9'37,32270’W, con una desviación estándar vertical de $0.01 \mathrm{~m}$ y horizontal de $0.006 \mathrm{~m}$ con lo que se puede identificar que es cercana a cero $(0)$ es decir, la dispersión de los datos es mínima (Spiegel, Schiller, Srinivasan \& Stephens, 2010). Además, se obtiene una precisión horizontal de $4.4 \mathrm{~cm}$ y vertical de $5.2 \mathrm{~cm}$, cumpliendo con el objetivo de determinar coordenadas geográficas con precisión centimétrica.

\section{ESTACIÓN CALARCÁ}

La estación acelerográfica Calarcá está ubicada en el municipio de Calarcá- Quindío específicamente en el corregimiento Gato Negro. La estación se encuentra al Sur-Este del centro poblado y cuenta con un acelerógrafo sobre roca, además del sistema de adquisición y de comunicaciones para recopilar la información y enviarla a las oficinas del observatorio sismológico de la Universidad del Quindío (Villalobos Nieto, Villa Zapata, \& Muñoz Loaiza 2008).

El equipo GPS no es posible instalarlo en el techo de la caseta por la abundante presencia de vegetación, por lo tanto, es necesario ubicarlo aproximadamente a 5 metros de la estación, teniendo en cuenta que este aspecto no influye de manera significativa en los resultados obtenidos, ya que se va a obtener una precisión centimétrica. Después de procesada la información, se obtienen las siguientes coordenadas georreferenciadas: 4³0’32,23290'N y 75³7’39,18501'W con una desviación estándar vertical de $0.015 \mathrm{~m}$ y horizontal de $0.008 \mathrm{~m}$, valores cercanos a cero (0) lo que quiere decir que la dispersión de los datos es muy pequeña. También se obtienen precisiones centimétricas, vertical de $3.1 \mathrm{~cm}$ y horizontal de $18.7 \mathrm{~cm}$, de lo cual se puede determinar que la precisión horizontal es menor debido a la distancia a la cual se ubica el receptor GPS (Abraira, 2002). 


\section{ESTACIÓN CAICEDONIA}

Caicedonia es un municipio del Valle del Cauca, ubicado al Oriente del departamento. En la zona rural de este municipio, se encuentra esta estación sismológica de registro perteneciente a la red del OSQ. El acceso a la estación, es posible realizarla en automóvil hasta cierta parte y un tramo se debe hacer caminando. Asi mismo, la estación está protegida por una reja que impide el paso de animales y personas, pues esta zona es paso obligado para llegar a algunas fincas del sector.

El tiempo de exposición del GPS es desde las 11:06am hasta las 2:14pm con una altura de antena de $1.71 \mathrm{~m}$. Las coordenadas obtenidas son: $4^{\circ} 19^{\prime} 10,91257^{\prime}{ }^{\prime} N$ y $75^{\circ} 48^{\prime} 50,73003$ 'W. Se tiene además una desviación estándar vertical de $0.00958 \mathrm{~m}$ y horizontal de $0.00731 \mathrm{~m}$, teniendo una dispersión de datos pequeña ya que tienden a cero (0). La precisión horizontal obtenida es de $3.8 \mathrm{~cm}$ y la vertical de $1.5 \mathrm{~cm}$, teniendo una precisión centimétrica muy buena.

\section{ESTACIÓN RIBERALTA}

La estación sismológica Riberalta está ubicada en el corregimiento Riberalta del Norte del Valle del Cauca, perteneciente al municipio de La Victoria, la cual se observa en la ilustración 2. El acceso a la estación se realiza en automóvil hasta donde termina el corregimiento, luego es necesario caminar alrededor de 20 minutos para llegar hasta donde está ubicada.

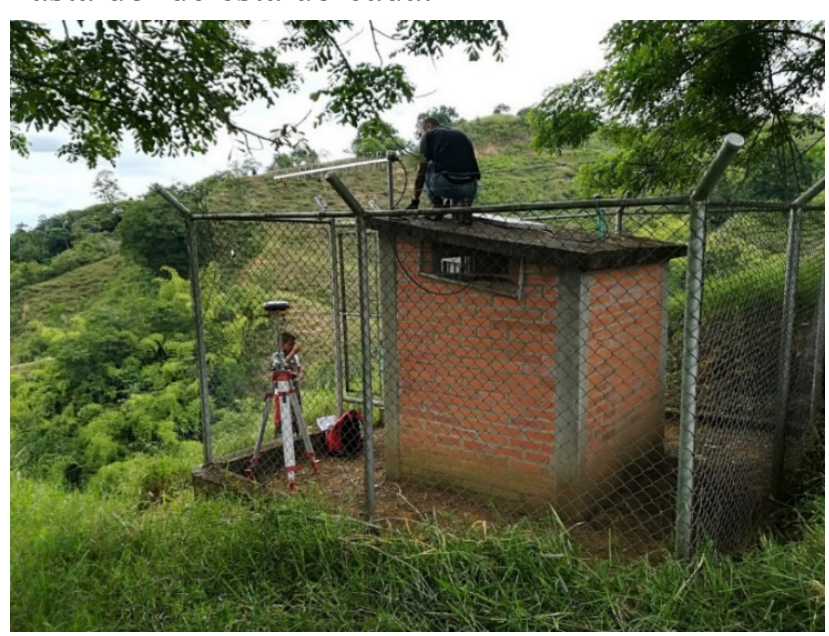

Ilustración 2.Estación Riberalta. Fuente: Propia
Las coordenadas geográficas determinadas son: 4³0'59,37819'N y 7553'12,90815'W. El GPS es posible ubicarlo al lado de la estación y por lo tanto se obtienen precisiones centimétricas con un tiempo de exposición de 2 horas: $1.4 \mathrm{~cm}$ horizontal y $2.6 \mathrm{~cm}$ verticalmente. Finalmente, se tiene una desviación estándar vertical de 0.022 $\mathrm{m}$ y horizontal de $0.015 \mathrm{~m}$, lo que se interpreta como una dispersión de los datos pequeña ya que tienden a cero $(0)$.

\section{ESTACIÓN PEÑAS BLANCAS}

En el ecoparque Peñas Blancas ubicado al Sureste del municipio de Calarcá, se encuentran las estaciones sismológicas del OSQ denominadas Peñas Blancas (PBLA), donde se cuenta con una tipo banda ancha y otra tipo periodo corto, constan de un sismómetro ubicado sobre roca, además de una antena transmisora, un panel solar, entre otros elementos.

Para llegar a esta estación, es posible realizar el acceso en vehiculo hasta una finca y desde ese lugar con la ayuda de un guía turístico se toma el camino hacia la peña ya que no existe la señalización adecuada para hacer el ascenso sin compañía.

Se realiza la instalación del equipo GPS muy cerca de la estación con lo cual se obtienen las siguientes coordenadas debidamente georreferenciadas: $75^{\circ} 37^{\prime} 54,15415^{\prime} \mathrm{W}$ y $4^{\circ} 28^{\prime} 47,41820^{\prime} \mathrm{N}$. Después de realizado el postproceso, se determina una precisión centimétrica horizontal de $1 \mathrm{~cm}$ y vertical de $1.7 \mathrm{~cm}$. A demás, la desviación estándar es vertical $0.036 \mathrm{~m}$ y horizontal de $0.009 \mathrm{~m}$ donde se identifica que tienden a cero (0) por lo tanto la dispersión de los datos es muy pequeña.

\section{ESTACIÓN MODÍN}

La estación sismológica Modín está ubicada en la zona rural del corregimiento Modín perteneciente al municipio de Cartago - Valle del Cauca. Para acceder al lugar, es necesario dejar el carro estacionado en una finca y atravesar caminando un terreno utilizado para la cría de ganado caballar para iniciar el ascenso a la montaña durante 20 
minutos aproximadamente. La caseta se encuentra protegida con una reja como se muestra en la ilustración 3 , la cual evita algún daño con el paso de personas y animales del sector.

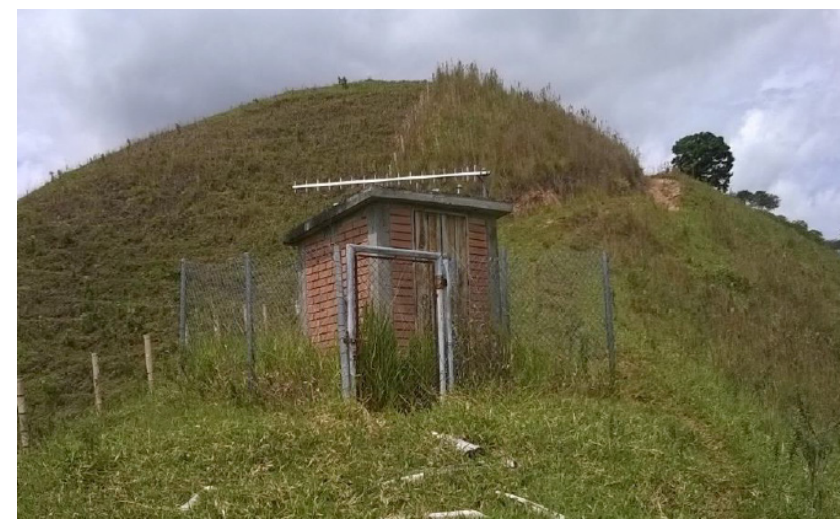

Ilustración 3. Estación Modín. Fuente: Propia.

En esta estación se encuentra un sismómetro sobre roca, un panel solar y la antena receptora está sobre la ladera aproximadamente a 5 metros de distancia, es en ese punto donde se instala el equipo GPS para la toma de datos. Las coordenadas georreferenciadas son: $75^{\circ} 52$ '53,80210"W y 440’35,39908'N. Se obtiene una precisión vertical de $1.9 \mathrm{~cm}$ y horizontal de $1.1 \mathrm{~cm}$, una desviación estándar vertical de $0.015 \mathrm{~m}$ y $0.008 \mathrm{~m}$ horizontal lo cual indica que la dispersión de la información recolectada es pequeña y muy precisa.

\section{ESTACIÓN BOLIVAR}

La estación sismológica Bolivar, aún no se encuentra en funcionamiento, pero muy pronto se tiene proyectado realizar las instalaciones necesarias para retomar el registro de eventos en la zona. Está ubicada en el municipio de Bolivar, en el embalse Sara Brut denominado tambien embalse de Guacas que es una de las principales fuentes de agua para consumo humano del norte del departamento del Valle del Cauca.

Las coordenadas geográficas determinadas son: $76^{\circ} 14$ '34,85158'"W y 4²0'28,37025'N , con una desviación estándar vertical de $0.030 \mathrm{~m}$ y horizontal de $0.012 \mathrm{~m}$ lo que indica que la dispersión de los datos tiende a cero (0). A demás, se tiene una precisión vertical de $3.2 \mathrm{~cm}$ y horizontal de $1.6 \mathrm{~cm}$, siendo la precisión centimetrica deseada para un tiempo de exposición de 2 horas y $30 \mathrm{mi}$ nutos.

\section{ESTACIÓN GÉNOVA}

La estación acelerográfica Génova, está ubicada en el municipio de Génova al sur del Quindío. Para llegar hasta el lugar, es necesario realizar un ascenso de 10 minutos desde la finca La Ermita. Esta estación cuenta con un acelerógrafo sobre roca, además de todos los instrumentos necesarios para garantizar su correcto funcionamiento. Presenta obstrucción visual en la parte de atrás de la caseta y por tal motivo el montaje del GPS se realiza en el techo para tener mejor recepción de los satélites.

Después de realizado el postproceso, se obtienen las siguientes coordenadas geográficas: $75^{\circ} 47^{\prime} 13,85084^{\prime \prime} \mathrm{W}$ y $4^{\circ} 14^{\prime} 47,70858^{\prime \prime} \mathrm{N}$. A demás, del ajuste de los datos se determina una desviación estandar vertical de $0.016 \mathrm{~m}$ y horizontal de $0.008 \mathrm{~m}$, lo que indica que la dispersión de los datos es pequeña. Las precisiones vertical y horizontal logradas durante un tiempo de exposición de 2 horas y 30 minutos son de $1,6 \mathrm{~cm}$ y $0,9 \mathrm{~cm}$ respectivamente.

\section{CONCLUSIONES}

Se planificaron las campañas de medición para la recolección de los datos en campo con el receptor GPS doble frecuencia de alta precisión South S82, utilizando el software GPS Planning Online, lo que representó una gran ayuda para identificar la disponibilidad de satélites y hacer más efectivas y precisas las mediciones realizadas en cada una de las estaciones de la red del Observatorio Sismológicos de la Universidad del Quindío (OSQ).

Se implementa el sistema de posicionamiento GNSS aplicando el método Postproceso mediante el software libre Topcon Tools, logrando realizar la debida georreferenciación de las coordena- 
das de las estaciones de registro con el respectivo ajuste de los datos para obtener mayor precisión en la localización futura de los eventos sísmicos registrados en la zona las cuales se pueden observar en la tabla 1. Este insumo tiene mucha im- portancia para la comunidad científica en general y específicamente para el personal del OSQ que son los encargados de interpretar las señales recibidas de las estaciones sismológicas y acelerográficas y localizar dichos eventos.

Tabla 1. Coordenadas de las estaciones de registro del OSQ.

\begin{tabular}{|c|c|c|c|}
\hline \multirow{2}{*}{$\begin{array}{c}\text { ESTACIONES DE REGISTRO DEL } \\
\text { OSQ }\end{array}$} & \multirow{2}{*}{ TIPO DE ESTACIÓN } & \multicolumn{2}{|c|}{ COORDENADAS GEORREFERENCIADAS } \\
\hline & & LONGITUD & LATITUD \\
\hline UNIQUINDIO & Acelerográfica (suelo) & 75³9'37,32270”'W & $4^{\circ} 33^{\prime} 21,83385^{\prime \prime} \mathrm{N}$ \\
\hline CALARCÁ & Acelerográfica (roca) & $75^{\circ} 37^{\prime} 39,18501^{\prime \prime} \mathrm{W}$ & $4^{\circ} 30^{\prime} 32,23290 ” \mathrm{~N}$ \\
\hline CAICEDONIA & Sismológica (roca) & $75^{\circ} 48^{\prime} 50,73003^{\prime \prime} \mathrm{W}$ & $4^{\circ} 19^{\prime} 10,91257^{\prime \prime} \mathrm{N}$ \\
\hline RIBERALTA & Sismológica (roca) & $75^{\circ} 53^{\prime} 12,90815^{\prime \prime} \mathrm{W}$ & $4^{\circ} 30^{\prime} 59,37819^{\prime \prime} \mathrm{N}$ \\
\hline PEÑAS BLANCAS & Sismológica (roca) & $75^{\circ} 37^{\prime} 54,15415^{\prime \prime} \mathrm{W}$ & $4^{\circ} 28^{\prime} 47,41820^{\prime \prime} \mathrm{N}$ \\
\hline MODÍN & Sismológica (roca) & $75^{\circ} 52^{\prime} 53,80210^{\prime \prime} \mathrm{W}$ & $4^{\circ} 40^{\prime} 35,39908^{\prime} \mathrm{N}$ \\
\hline BOLIVAR & Sismológica (roca) & $76^{\circ} 14^{\prime} 34,85158^{\prime \prime} \mathrm{W}$ & $4^{\circ} 20^{\prime} 28,37025^{\prime \prime} \mathrm{N}$ \\
\hline GÉNOVA & Acelerográfica (roca) & $75^{\circ} 47^{\prime} 13,85084^{\prime \prime} \mathrm{W}$ & $4^{\circ} 14^{\prime} 47,70858^{\prime \prime} \mathrm{N}$ \\
\hline
\end{tabular}

Este proyecto sirve como complemento de la investigación realizada en el año 2016 por Juan Esteban Gonzales Jaramillo titulada: Mapeo de las rutas de acceso a las estaciones de medición del Observatorio Sismológico de la Universidad del Quindío en el cual generó mapas base para facilitar el trayecto a cada estación de la red del OSQ (González Jaramillo, 2016).

Finalmente, se crea un mapa con las coordena- das geográficas debidamente georreferencias de las estaciones sismológicas y acelerográficas del Observatorio Sismológico de la Universidad del Quindío que se puede apreciar en la figura 2. Cabe resaltar que el centro de monitoreo del OSQ donde se realizó el procesamiento de la información, está ubicado cerca a la estación acelerográfica UQ y por consiguiente a la estación de referencia REF-UQ, razón por la cual, no se representa en el mapa debido a la proporción de la escala. 


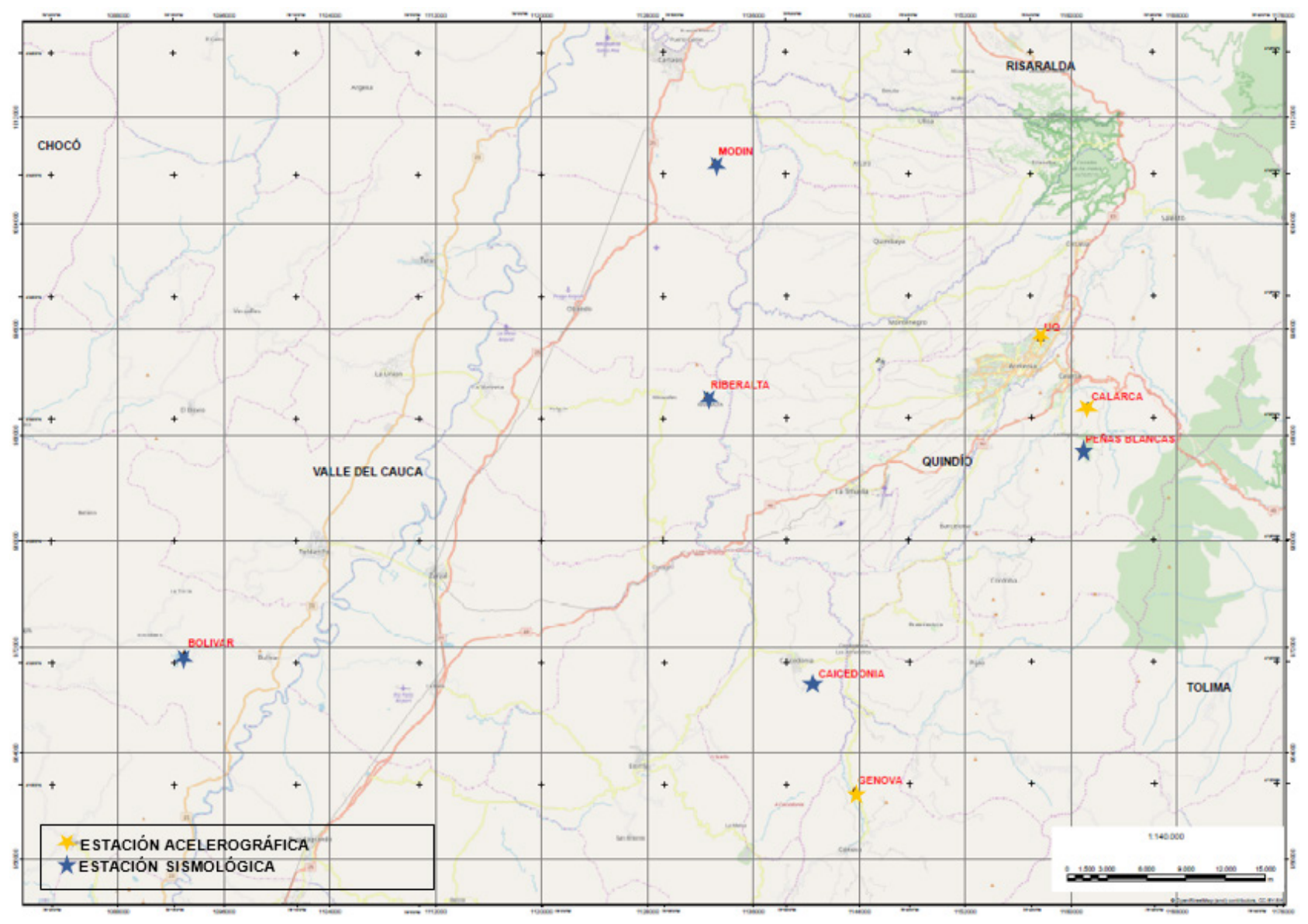

Figura 2. Ubicación de las estaciones de medición del OSQ georreferenciadas. Fuente: Propia realizada en ArcGIS

\section{AGRADECIMIENTOS}

Al Observatorio Sismológico de la Universidad del Quindío que nos brindó el espacio para desarrollar este proyecto.
Al laboratorio de topografía de la Universidad del Quindío y al ingeniero Alejandro Blandón por facilitarnos algunos dispositivos usados y su conocimiento sobre el tema.

\section{BIBLIOGRAFÍA}

1. Abraira, V. (2002). Desviación Estándar y error estándar. SEMERGEN-Medicina de Familia, 28(11), 621.

2. ArcGis. (9 de Noviembre de 2013). Georrerenciación y Sistemas de Coordenadas [Sitio web]. Recuperado el 16 de marzo de 2018, de https://bit.ly/2fYylzZ

3. Ghilan, C. D. (2010). Adjustment of GNSS Networks. En C. D. Ghilan, Adjustment Computations. Spatial Data Analysis (págs. 327-352). New Jersey: John Wiley \& Sons, Inc.

4. González Jaramillo, J. E. (2016). Mapeo de las rutas de acceso a las estaciones de medición del Observatorio Sismológico de la Universidad del Quindío. Armenia.

5. McCormac, J. (2008). Topografia. Michigan: Limusa Wiley.

6. Pachas, R. (2010). Posicionamiento estático diferencial: errores y postproceso. Trujillo, Venezuela.

7. Spiegel, M., Schiller, J., Srinivasan, A., \& Stephens, M. (2010). Probabilidad y Estadística. México: Mc Graw Hill.

8. Topcon Tools. (2017). Procesamiento de Datos Vr 8.2.3 [Software Libre]. Bogotá.

9. Villalobos Nieto, G., Villa Zapata, D., \& Muñoz Loaiza, A. (2008). El Sistema Mundial de Navegación por Satélite. En Geodesia espacial y sus aplicaciones (pág. 44). Armenia- Quindío: Elizcom. 